\title{
Dual inflow, total-arterial, anaortic, off-pump coronary artery bypass grafting: how to do it
}

\author{
Fabio Ramponi ${ }^{1,2,3}$, Michael Seco ${ }^{1,2,3}$, James B. Edelman ${ }^{1,2,3,4}$, Andrew G. Sherrah ${ }^{1,3,4}$, Paul G. Bannon ${ }^{1,2,4,5}$, R. \\ John L. Brereton', Michael K. Wilson ${ }^{1,3,6,7}$, Michael P. Vallely ${ }^{1,2,3,5,7}$ \\ ${ }^{1}$ Cardiothoracic Surgical Unit, Royal Prince Alfred Hospital, Sydney, Australia; ${ }^{2}$ The University of Sydney, Sydney, Australia; ${ }^{3}$ Coronary Artery \\ Disease Institute, Sydney, Australia; ${ }^{4}$ The Baird Institute of Applied Heart and Lung Surgical Research, Sydney, Australia; ${ }^{5}$ Sydney Heart and Lung \\ Surgeons, Sydney, Australia; ${ }^{6}$ Cardiothoracic Surgical Unit, Royal North Shore Hospital, Sydney, Australia; ${ }^{7}$ Macquarie University Hospital, Sydney, \\ Australia \\ Correspondence to: Prof. Michael P. Vallely, PhD, FRACS. Sydney Heart and Lung Surgeons, 209/100 Carillon Avenue, Newtown NSW 2042, \\ Australia. Email: michael.vallely@sydneyheartandlung.com.au.
}

\begin{abstract}
Coronary surgery performed on an arrested heart, using one internal mammary artery and a saphenous vein carries two main potential drawbacks: the known failure rate of vein grafts and the relatively high rate of neurologic injury. To address these concerns, we describe a technique that achieves complete revascularization without manipulating the ascending aorta (anaortic, off-pump) and utilizing total arterial grafts. All patients undergo thorough preoperative investigation, including bilateral carotid, vertebral and subclavian artery Duplex ultrasounds. A pulmonary artery catheter, transoesophageal echocardiography, and point-of-care coagulation testing are used in each case. The left and right internal mammary arteries and non-dominant radial artery are harvested using a fully skeletonised technique. Wide bilateral extrapleural retrothymic tunnels are developed and the pericardium is opened widely to facilitate cardiac positioning. A tandem graft is constructed with the right internal mammary artery (RIMA) in situ and radial artery using an end-to-end anastomosis. This graft is brought into the pericardium and through the transverse sinus in order to graft the lateral and inferior walls with multiple sequential distal anastomoses. The left internal mammary artery (LIMA) in situ is used to graft the anterior wall. Four main cardiac positions (high and low lateral walls, inferior and anterior walls) are obtained using a combination of off-pump stabilizer positioning, alternate tension on pericardial 'heart-strings', table tilting and folded wet sponges. All distal anastomoses are performed using silastic intracoronary shunts and an off-pump myocardial stabilizer. All grafts are checked using transit-flow time measurements. Milrinone is continued overnight and dual antiplatelet therapy is continued for 3 months postoperatively.
\end{abstract}

Keywords: Coronary artery bypass; radial artery; mammary arteries; aorta; stroke

Submitted Feb 12, 2018. Accepted for publication Jun 20, 2018.

doi: 10.21037/acs.2018.06.17

View this article at: http://dx.doi.org/10.21037/acs.2018.06.17

\section{Introduction}

The most widely performed surgical coronary revascularization technique remains the left internal mammary artery (LIMA) to the left anterior descending (LAD) artery and reversed long saphenous vein to other arteries, performed using cardiopulmonary bypass on an arrested heart. However, there are two potentially significant drawbacks of this technique, relating to graft failure and neurological injury.

Firstly, long saphenous vein grafts have well-known early and late failure rates (1). This contrasts with established evidence of long term survival benefit from the addition of a right internal mammary artery (RIMA) to both the left and right coronary systems (2) as well as the use of multiple arterial grafting strategies (3).

The second drawback is the relatively high rate of 
neurological injury, particularly in high-risk subgroups $(4,5)$. Indeed, in the SYNTAX trial the major criticism of the coronary artery bypass grafting (CABG) arm was the increased risk of periprocedural stroke as compared to percutaneous coronary intervention (PCI) $(2.2 \%$ CABG vs. $0.6 \%$ PCI; $\mathrm{P}=0.003$ ) (6). In a large network meta-analysis performed by our group, the elimination of all aortic manipulation during CABG was shown to significantly reduce the risk of stroke (7) and avoiding cardiopulmonary bypass also reduced overall risks of early mortality and morbidity (7).

In recent times the incidence and negative impact of more subtle neurocognitive injury and psychiatric issues following CABG has also been recognized (8). The aetiology has been linked to silent brain infarcts, cerebral hypoperfusion, microemboli and a systemic inflammatory response, which are often associated with cardiopulmonary bypass (9). Numerous ongoing studies are investigating whether avoidance of aortic manipulation and cardiopulmonary bypass improves outcomes in this group as well.

Critics of off-pump coronary artery bypass grafting (OPCABG) argue that increased technical difficulty of the technique may result in reduced graft patency and reduced graft numbers, leading to incomplete surgical revascularization and worse long term survival $(10,11)$. The results of the ROOBY trial comparing off- $v s$. on-pump CABG, performed by relatively inexperienced OPCABG surgeons (20 eligible cases, $55 \%$ performed by residents), has demonstrated reduced 5 -year survival for patients undergoing OPCABG (12). However, this is not the case in groups that have high-volume experience and expertise in OPCABG surgery, as the 5-year CORONARY trial data have demonstrated (13).

To address these concerns we describe a simple and reproducible technique to accomplish complete revascularization without manipulating the ascending aorta (anaortic) utilizing total arterial grafts. The LIMA is anastomosed the LAD and the RIMA extended with the radial artery as a tandem graft via the transverse sinus to the lateral and inferior walls.

\section{Operative techniques}

\section{Preoperative assessment and investigations}

All patients referred for CABG undergo routine blood tests, electrocardiography, chest X-ray imaging, spirometry and a transthoracic echocardiogram to assess for valvular disease and ventricular function and dilatation. All patients also undergo bilateral carotid, vertebral and subclavian artery duplex scans to assess concomitant peripheral vascular disease and suitability of the internal mammary arteries for inflow. If significant carotid or subclavian disease is detected, then patients undergo a CT aortogram with 4-vessel runoff to assess the arch vessel branches and the Circle of Willis. The suitability of the radial artery is assessed with an Allen's test ( $<5$ seconds deemed suitable) (14). In borderline cases, a pulse oximeter is used to perform the Allen's test and if a pulsatile wave-form is obtained, then the artery is harvested, otherwise an alternative conduit is used. Aspirin is continued up to the time of surgery.

\section{Anaesthetic considerations}

Maintenance of normothermia throughout the operation is important and active warming with a forced-air warming blanket is commenced on arrival to the anaesthetic room. All patients have a Swann-Ganz pulmonary artery catheter inserted and a transoesophageal echocardiogram (TOE) is performed. Elevation of the pulmonary artery diastolic (PAD) pressure in the presence of hypotension and regional wall motion abnormalities respectively are sensitive indicators of ischaemia.

The patient is commenced on low dose intravenous milrinone $(0.1-0.2 \mu \mathrm{g} / \mathrm{kg} / \mathrm{min})$, without a loading dose, after induction of anaesthesia. This has a two-fold benefit in increasing cardiac output for end-organ perfusion (15) and as a systemic vasodilator when using all arterial grafts (16). Avoiding a loading dose of milrinone may reduce haemodynamic instability (17). Patients usually require a background noradrenaline infusion $(0.01-0.2 \mu \mathrm{g} / \mathrm{kg} / \mathrm{min})$. The use of intravenous beta-blockers (metoprolol $2.5-5 \mathrm{mg}$ boluses) is often required to achieve an optimal heart rate of 60-70 bpm.

Maintaining normovolaemia is important, as hypovolaemia is poorly tolerated particularly during repositioning of the heart. TOE and observation of cardiac filling by the surgeon is useful to guide volume status, together with arterial systolic pressure variation and dynamic responses of central venous pressure and PAD pressure to fluid boluses. Intravascular volume is maintained with synthetic colloids and intracellular volume is maintained with a background maintenance crystalloid infusion. Systemic heparinization is used to maintain an ACT greater than 450 seconds. A cell-saver is used for all cases. Point-of-care coagulation testing is now performed 


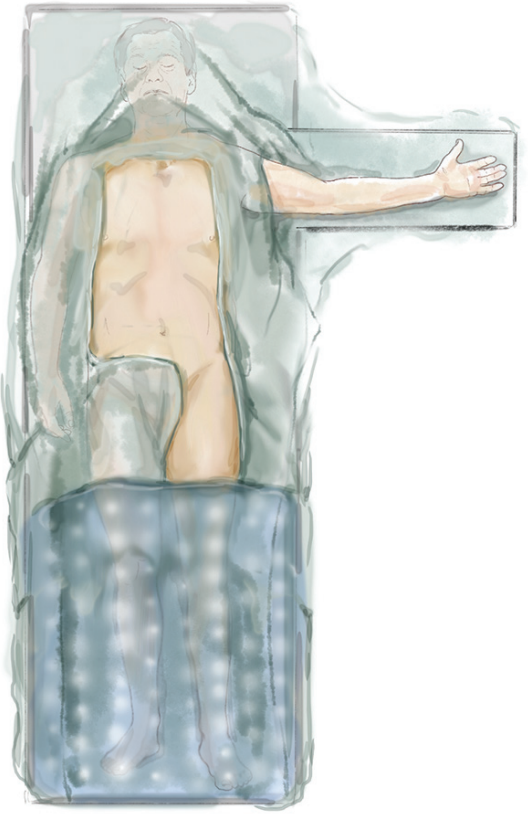

Figure 1 Patient positioning with non-dominant arm abducted to 90 degrees for simultaneous radial artery harvest during internal mammary artery harvest. The legs are covered with a warming blanket to help maintain normothermia.

routinely at our centre using rotational thromboelastometry (ROTEM) and multiplate platelet function analysis.

The patient is positioned supine with the left arm gently abducted on an arm-board (Figure 1). He or she is prepped and draped with a forced-air warming blanket covering the lower limbs.

\section{Conduit and graft configurations}

Our standard operation is to revascularize the LAD with an in situ skeletonized LIMA. The lateral and inferior walls are revascularized with an in situ skeletonized RIMA/radial tandem graft brought via the transverse sinus (Figure 2). This separates the anterior wall blood supply from the rest of the heart, thus protecting the integrity of the LIMA to LAD graft. There are also no grafts crossing the midline anteriorly enabling safe management of mediastinitis and redo surgery. Performing multiple skip grafts using the radial artery is technically easier than using a RIMA, a view shared by others (18).

Our aim is to utilize all arterial grafts. However, this is not always possible. Radial arteries may not be suitable (e.g., due to a negative Allen's test or dystrophic calcification) and

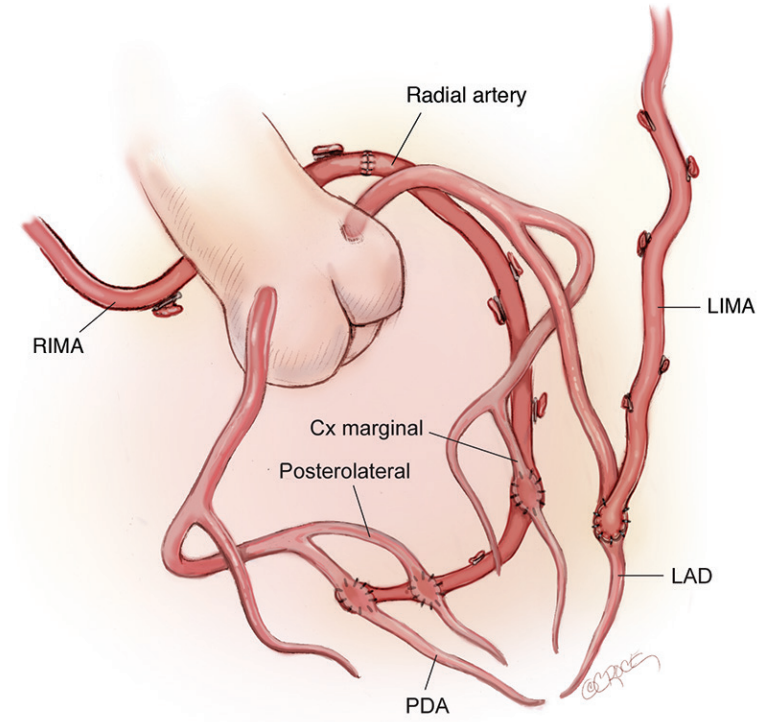

Figure 2 Configuration of grafts to achieve complete revascularization using a left internal mammary artery (LIMA) to left anterior descending (LAD) artery, and right internal mammary and radial artery tandem graft to lateral and inferior walls. PDA, posterior descending artery.

the patient's occupation (e.g., concert violinist) or personal wishes may dictate a thigh vein as the graft of choice to extend the RIMA. Rarely, the right gastro-epiploic artery may be used.

\section{Conduit harvesting and management}

The internal mammary arteries are harvested using a fully skeletonized technique (Figure 3). This minimizes chest wall trauma, achieves maximum length, and reduces the risk of deep sternal wound infection during bilateral internal mammary artery harvest (19). The LIMA is harvested first. The left sternal edge is retracted using a Rultract Skyhook (Rultract, Cleveland, USA) as this provides optimal exposure. The LIMA is exposed by pushing the pleura laterally. The endothoracic fascia is carefully incised directly below and along the length of the LIMA using long Dietrich's forceps and low power diathermy $(20 \mathrm{~W})$. The medial internal mammary vein is identified at the subclavian vein and divided after double clipping both sides, as this improves the final length and lie of the LIMA and enables safe proximal harvest. The LIMA is harvested from above the left subclavian vein to its bifurcation, using a combination of blunt and sharp diathermy dissection and 


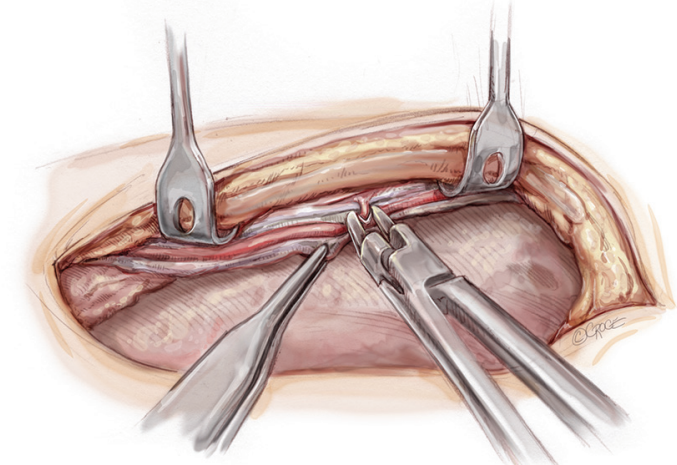

Figure 3 Left internal mammary artery harvest using a fully skeletonized technique.

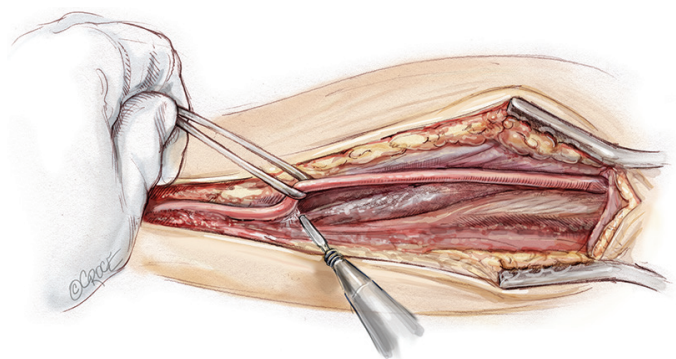

Figure 4 Radial artery harvest using an open, fully skeletonized technique. A soft silicon vessel-loop is used to gently retract the radial artery whilst low power diathermy is used to dissect and cauterize branches.

dividing the branches with diathermy after clipping the graft side. The RIMA is harvested using a similar technique; it is mobilized up to the subclavian vein, with the medial soft tissue swept back to the strap muscles to allow an unimpeded lie for the graft as it is brought medially. After systemic heparinization both IMAs are sprayed liberally with papaverine solution before being divided distally and then wrapped in a warm, papaverine soaked gauze. Length is not usually an issue with this operation and the RIMA can be divided proximal to the bifurcation to preserve lower sternal blood supply.

The radial artery is harvested from the non-dominant arm; an open, fully skeletonized technique provides increased length, allows all soft-tissue impediments to be removed to maximize conduit dilatation, and provides ease of use when performing distal anastomoses. A curvilinear incision, from above the wrist crease to below the elbow crease, is made on the volar aspect of the forearm utilizing the groove medial to brachioradialis. This incision provides optimal exposure of the radial artery, reduces the chance of injury to the medial and lateral cutaneous nerves of the forearm and provides the best cosmetic and functional result by reducing cicatrisation along the length of the wound.

With the diathermy on low power (15-20 W), the radial artery is exposed along its length between brachioradialis and flexor carpi radialis. The fascia over the artery is divided using Pott's scissors, skeletonizing the radial artery along its length between the recurrent radial and superficial palmar branches. The artery is then suspended using a silastic vessel-loop, which provides tension with minimal trauma and the branches are divided under tension with diathermy (Figure 4). Only briskly bleeding branches are controlled with a small hemoclip (the remaining branches are clipped after fashioning the tandem graft with the RIMA and obtaining maximal pharmacological and hydrostatic dilatation). The artery is then divided proximally and distally after double clipping the patient sides. A slit is subsequently made in the proximal end of the graft with Pott's scissors for proximal orientation and as preparation for the tandem graft anastomosis. The artery is then cannulated with a 2.0-mm silastic olive-tip cannula and the graft gently flushed through with $20 \mathrm{~mL}$ of heparinized blood containing $5 \mathrm{mg} / 50 \mathrm{~mL}$ of verapamil and left to soak in the same solution. The wound is closed over a small suction drain, dressed and the arm placed back against the body.

\section{Preparation of the pericardium}

Bilateral, extrapleural retrothymic tunnels are developed before opening the pericardium. Dissection is easier with the pericardium intact, as retraction is maintained. The leftsided tunnel is prepared by mobilizing the thymic remnant and fat-pad off the aortic arch. Using a finger, the superior entry to the tunnel is dissected below the innominate vein. This tunnel needs to be wide to prevent kinking of the LIMA and to ensure that the lie is optimal. The right retrothymic tunnel is made by dissecting the thymic fat pad off the pericardium down to the superior vena cava (SVC). The tunnel is then mobilized superiorly and medial to the lung in an extrapleural fashion. There are often bands of connective tissue with accompanying veins that need dividing to allow an unimpeded path for the graft into the retrothymic tunnel. The RIMA is not brought through the tunnel until after the tandem graft is constructed.

The pericardium is opened longitudinally with the left sided pericardium opened laterally at the diaphragm and 


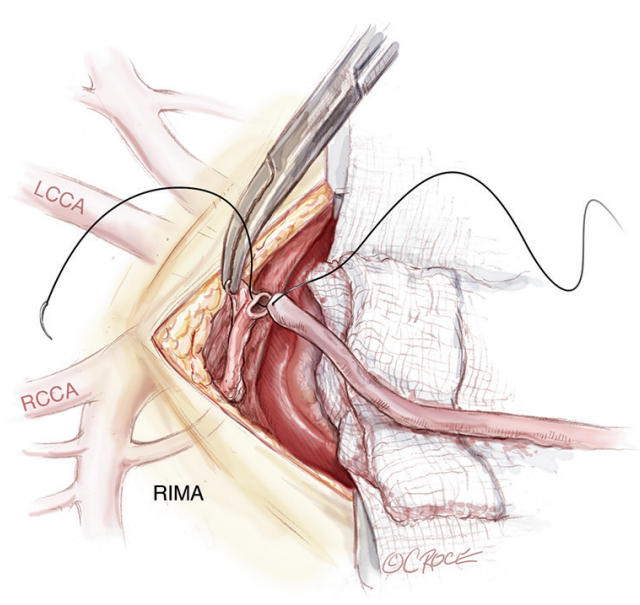

Figure 5 Right internal mammary artery to radial artery end-toend anastomosis. LCCA, left common carotid artery; RCCA, right common carotid artery; RIMA, right internal mammary artery.

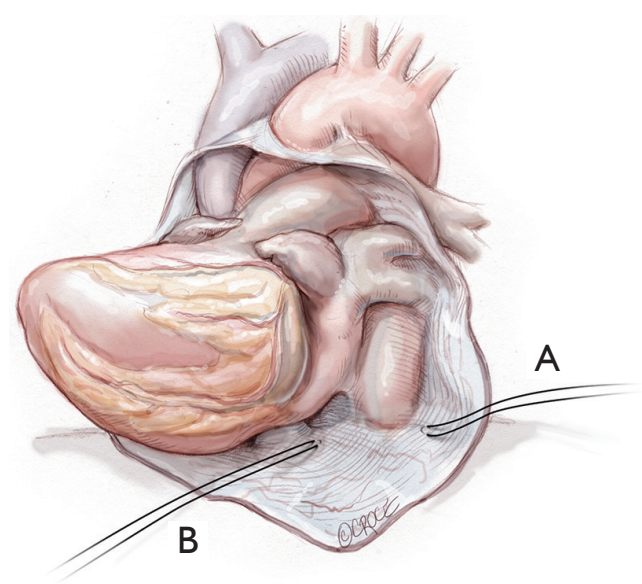

Figure 6 Pericardial heart strings: (A) left lateral inferior pericardial recess; (B) diaphragmatic surface medial to the IVC. IVC, inferior vena cava.

obliquely at the pulmonary valve. The proximal oblique incision is important to allow the LIMA ease of entry into the pericardium without compromising the lie of the graft. The left pericardial flap is then hitched up on the sternum, behind the sternal retractor. The right pericardium is then incised down to the inferior vena cava (IVC) at the diaphragm and at the superior atriocaval junction. This allows for exposure of the lateral wall vessels by rolling the heart on the caval axis into the right pleural space, providing maximum exposure with minimal effect on venous return. Extreme care must be taken not to injure the phrenic nerve.
The distal targets are then assessed.

\section{Preparation of the RIMA/radial tandem graft}

A cradle is made with a medium dry sponge and a folded wet sponge, suspended over the heart with artery clips. This allows optimal conditions for the careful fashioning of the tandem graft. All arterial anastomoses are performed using a Prolene (polypropylene) 'flat-pack' $60 \mathrm{~cm} \mathrm{7-0}$ suture with an $8.0 \mathrm{~mm}$ needle (Ethicon, Somerville, United States). This allows maximum flexibility for parachuting anastomoses with the benefit of minimal 'memory' in the suture as well as advantages of a smaller, sharper needle without the difficulty associated with 8-0 Prolene. The RIMA is then attached to a folded wet sponge placed at the superior aspect of the sternal wound (Figure 5). The RIMA and radial artery are bevelled prior to fashioning the endto-end anastomosis. This serves to compensate for any size mismatch between the conduits and to prevent pursestringing and narrowing at the anastomosis.

The anastomosis is tightened and tied down after release of the bull-dog clamp. This allows one to maximize anastomosis tension in order to prevent leaks without causing purse-stringing. If there is any residual vasospasm the tandem graft is flushed with heparin and verapamilinfused blood via the radial artery and the outside of the conduit is sprayed with neat papaverine solution. Care is taken to release any soft-tissue bands across the arterial grafts. The radial artery side branches are then clipped with small hemoclips.

\section{Cardiac positioning and grafting}

Two pericardial 'heart strings' (1 Nylon) are placed at the following positions (Figure 6):

(I) The left, lateral, inferior pericardial recess;

(II) Diaphragmatic surface, medial to the IVC.

Care must be taken not to injure major structures deep to the pericardium. The left atrial appendage is inspected and is usually resected with a linear cutting Extra Thick Endo GIA Tri-Staple stapler (Medtronic, New Haven, USA) (Figure 7). This facilitates passage of the tandem graft via the transverse sinus and grafting of intermediate or high obtuse marginal coronary arteries. Resecting the left atrial appendage may also reduce the risk of stroke related to atrial fibrillation in the perioperative period (20). An Octopus Evolution heart stabilizer (Medtronic, Minneapolis, USA) is used to stabilize the heart when resecting the appendage. 


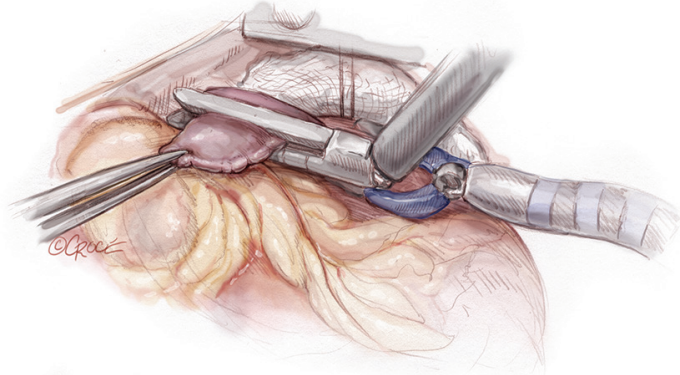

Figure 7 Left atrial appendage resection using an extra thick linear cutting stapler. The off-pump stabilizer is used to stabilize the heart during the resection. The staple line is inspected carefully for bleeding.

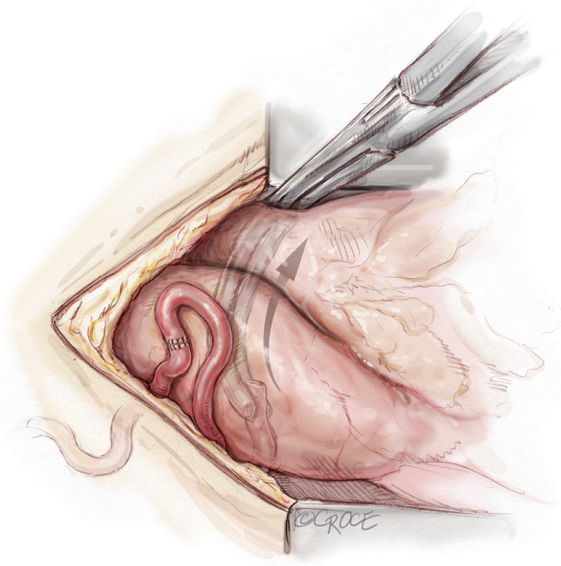

Figure 8 A Semb clamp is used to pass the right internal mammary to radial artery tandem graft through the transverse sinus.

The staple line is inspected and carefully cauterized with diathermy to prevent bleeding.

The RIMA/radial tandem graft is then brought behind the right thymic fat pad and over the SVC into the pericardium. The graft is subsequently brought via the transverse sinus to the left side of the heart with a Semb clamp passed left to right via the transverse sinus and grasping the tip of the graft (Figure 8). The heart is then placed in its natural position before gently pulling the graft through the transverse sinus. This is to remove any tension or traction on the graft and to prevent clip dislodgement. If the graft is impeded within the transverse sinus, it is easily mobilized by using a finger to free any loose adhesions, before pulling it out to length. Correct orientation is then checked by visual inspection.

The Octopus Evolution stabilizer is used to stabilize the heart for grafting. Warm and wet sponges and adjustment of the operating table position are utilized to obtain optimal surgical access and visualization. Regular communication with the anaesthetic staff, boluses of intravenous fluid, and judicious use of beta blockers and vasoconstrictors, combined with subtle re-adjustments of cardiac position are often required to obtain the best haemodynamic and operative conditions.

There are four major cardiac positions used for grafting (Figure 9), as outlined below:

(I) High-lateral wall vessels ( $1^{\text {st }}$ diagonal, intermediate, $1^{\text {st }}$ obtuse marginal): the table is rolled towards the surgeon with the patient's head down. Heart string 1 is placed on tension with wet sponges behind the heart as required. The heart is rolled across and vertically towards the right chest.

(II) Low-lateral wall vessels $\left(2^{\text {nd }}\right.$ and $3^{\text {rd }}$ marginal, posterolateral circumflex): the table remains in the same position as above. Heart string 1 is tightened across the arm of the sternal retractor, with Heart string 2 pulled inferiorly. The stabilizer is moved down to the target vessel with the heart remaining in the right chest.

(III) Inferior wall vessels (posterolateral right and right posterior descending): the heart is positioned 'apexup'. The table is centered, remaining head-down. Heart string 2 is used, with a wet pack placed medial to the IVC. If the heart moves across to the left, lateral tension on Heart string 1 and placement of an additional wet sponge against the lateral wall can help to prevent this.

(IV) Anterior wall vessels ( $2^{\text {nd }}$ and $3^{\text {rd }}$ diagonal, LAD): often a wet pack behind the heart is all that is required. Some tension on Heart string 1 laterally can be helpful.

It is not necessary to impart excess length to the grafts, as the grafts will loosen when the sternum is re-approximated. In fact, this can be detrimental and may cause kinking of the graft between sequential anastomoses. To provide optimal lie for the RIMA/radial tandem graft, it is important to assess the location of each distal anastomosis before commencing. Marking the graft and coronary artery with a surgical marking pen can be useful.

The anastomoses are completed in the order of the four positions listed above (high-lateral wall first, anterior wall last). Using the RIMA/radial tandem graft, longitudinal sequential side-to-side anastomoses are performed on the high and low-lateral walls, and perpendicular sequential 
A

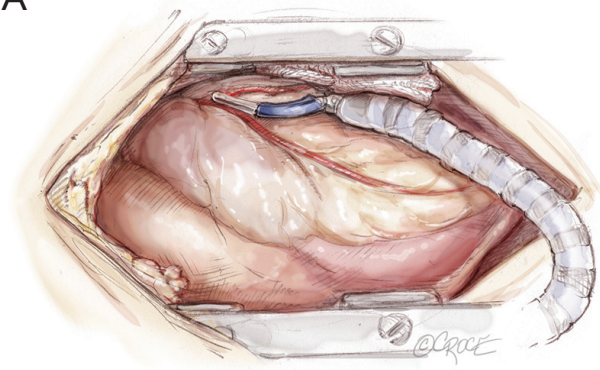

C

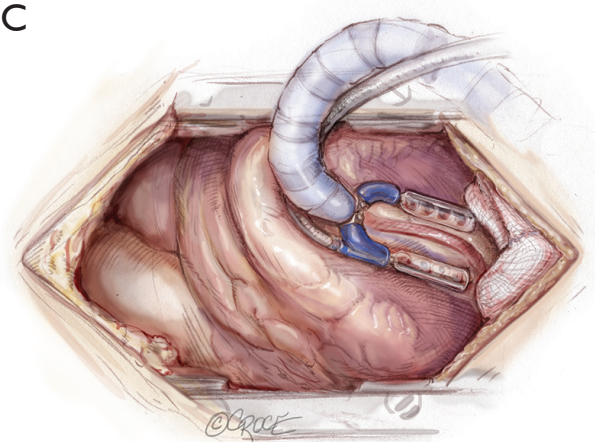

B

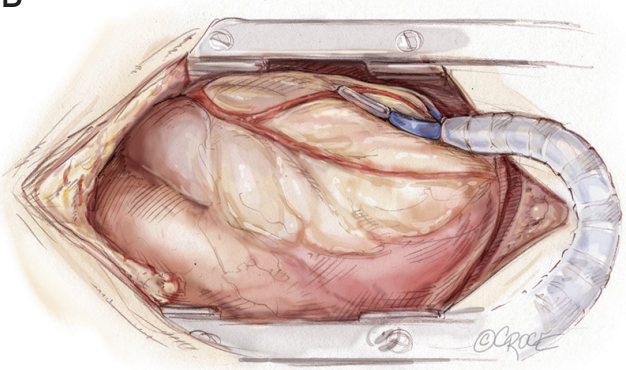

D

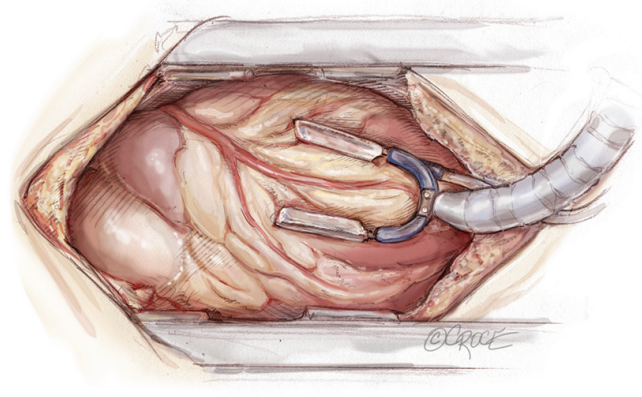

Figure 9 Four major cardiac positions: (A) high lateral wall vessels; (B) low-lateral wall vessels; (C) inferior wall vessels; (D) anterior wall vessels.

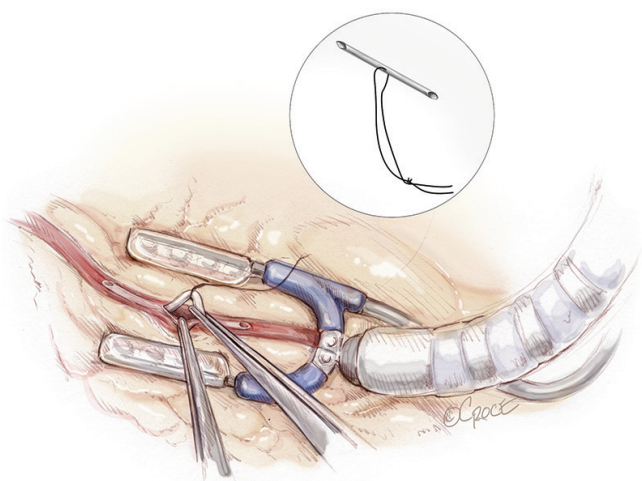

Figure 10 Home-made, silastic intra-coronary shunts are used to facilitate the off-pump anastomoses.

side-to-side anastomoses are formed on the inferior wall. After completion of the last anastomosis on the inferior wall, the end of the graft is clipped and secured to the epicardium with a 7-0 Prolene suture. On the anterior wall, an end-to-side anastomosis is used for the LAD artery.

Silastic intracoronary shunts are used when performing all distal anastomoses. These are 'home-made' with a 5-0 silk suture passed through the shunt and clipped with a small hemoclip (Figure 10) (21). The length is adjusted according to the diameter of the coronary artery. Silastic slings or bulldogs are used sparingly in an effort to reduce inadvertent injury to the coronary artery or to the ventricular wall. If a sling is required then it is not tightened until the shunt is placed in order to reduce coronary endothelial injury. Shunts provide a bloodless field, prevent technical errors such as catching the back wall of the coronary artery with a suture and provide some distal coronary artery flow during grafting. We prefer the silastic shunts to commercially available shunts because the 7-0 Prolene suture can be easily pulled through the shunt if it is caught during grafting.

After completion of the last distal anastomosis, all are checked visually for bleeding. Transit-time flow measurement is then performed using Medistim (Medistim, Oslo, Norway) to assess graft patency. Acceptable parameters for a left-sided graft are a mean graft flow of $\geq 15 \mathrm{~mL} / \mathrm{min}$, pulsatility index of $\leq 3.0$ and diastolic flow $>50 \%$; however, these need to be interpreted in the context of target vessel size, stenosis severity and graft diameter. A detailed discussion of the utility and application of Medistim is covered in other articles (22).

Heparin is fully reversed with protamine sulfate. The pericardium is then partially or fully apposed. This provides 
cardiac and graft coverage in the event of mediastinitis or if re-do surgery is required at a later date. The graft flows are checked again by applying the Medistim probe proximally on each side above the thymus. This is to ensure that the pericardial apposition has not compromised graft flow.

Epicardial pacing wires are not used routinely. A 24-Fr Blake drain (Ethicon, Somerville, United States) is placed in each pleural space and in the mediastinum. The Blake drains stay in situ until the morning of postoperative day 2 (or longer if required) in an effort to reduce the need for postoperative pleural effusion drainage. The milrinone infusion is continued overnight until the morning of postoperative day 1 . This provides vasodilation of the arterial grafts as well as increased cardiac output for endorgan perfusion. Calcium channel antagonists are not used. All patients receive aspirin and clopidogrel as soon as postoperative bleeding has settled ( $<100 \mathrm{~mL} / \mathrm{hr})$. Clopidogrel is continued for 3 months postoperatively (23).

\section{Beating heart on-pump}

It is possible to completely revascularize all patients without cross-clamping the ascending aorta. However, some patients may require cardiopulmonary bypass support to achieve this. Ideally, this is performed in an elective manner and there are several clinical scenarios that may make this more likely. For example, patients with large hearts, recent ischaemic instability or severe left main coronary artery disease are more likely to require circulatory support when performing the grafts. In these cases we have a primed cardiopulmonary bypass machine in the operating theatre. If assessment of the aorta with TOE and epiaortic ultrasound demonstrates no abnormalities then the ascending aorta is used for arterial inflow (24). However, if the ascending aorta is not suitable, then the right axillary artery is used for inflow. If the left ventricle distends during positioning, then a right superior pulmonary vein vent is placed. An epicardial stabilizer and intracoronary shunts are used as described above.

\section{Comments}

In an era of increasingly high risk patients and the continued development of percutaneous alternatives, a standardized CABG technique that improves both shortterm complications and long-term survival is necessary. In this paper we have described a reproducible technique for performing surgical revascularization that avoids cardiopulmonary bypass, avoids all manipulation of the ascending aorta, uses all-arterial grafts to achieve complete revascularization and separates anterior wall inflow from other territories for maximum performance.

\section{Acknowledgements}

None.

\section{Footnote}

Conflicts of Interest: The authors have no conflicts of interest to declare.

\section{References}

1. Gaudino M, Antoniades C, Benedetto U, et al. Mechanisms, Consequences, and Prevention of Coronary Graft Failure. Circulation 2017;136:1749-64.

2. Benedetto $U$, Caputo $M$, Gaudino $M$, et al. Is the right internal thoracic artery superior to saphenous vein for grafting the right coronary artery? A propensity scorebased analysis. J Thorac Cardiovasc Surg 2017;154:12691275.e5.

3. Locker C, Schaff HV, Dearani JA, et al. Multiple arterial grafts improve late survival of patients undergoing coronary artery bypass graft surgery: analysis of 8622 patients with multivessel disease. Circulation 2012;126:1023-30.

4. Roach GW, Kanchuger M, Mangano CM, et al. Adverse cerebral outcomes after coronary bypass surgery. Multicenter Study of Perioperative Ischemia Research Group and the Ischemia Research and Education Foundation Investigators. N Engl J Med 1996;335:1857-63.

5. Puskas JD, Thourani VH, Kilgo P, et al. Off-pump coronary artery bypass disproportionately benefits highrisk patients. Ann Thorac Surg 2009;88:1142-7.

6. Mohr FW, Morice MC, Kappetein AP, et al. Coronary artery bypass graft surgery versus percutaneous coronary intervention in patients with three-vessel disease and left main coronary disease: 5-year follow-up of the randomised, clinical SYNTAX trial. Lancet 2013;381:629-38.

7. Zhao DF, Edelman JJ, Seco M, et al. Coronary Artery Bypass Grafting With and Without Manipulation of the Ascending Aorta: A Network Meta-Analysis. J Am Coll Cardiol 2017;69:924-36.

8. Indja B, Seco M, Seamark R, et al. Neurocognitive and Psychiatric Issues Post Cardiac Surgery. Heart Lung Circ 
2017;26:779-85.

9. Seco M, Edelman JJB, Van Boxtel B, et al. Neurologic Injury and Protection in Adult Cardiac and Aortic Surgery. J Cardiothorac Vasc Anesth 2015;29:185-95.

10. Garcia S, Sandoval Y, Roukoz H, et al. Outcomes after complete versus incomplete revascularization of patients with multivessel coronary artery disease: a meta-analysis of 89,883 patients enrolled in randomized clinical trials and observational studies. J Am Coll Cardiol 2013;62:1421-31.

11. Mocanu V, Buth KJ, Kelly R, et al. Incomplete revascularization after coronary artery bypass graft operations is independently associated with worse longterm survival. Ann Thorac Surg 2014;98:549-55.

12. Hattler B, Messenger JC, Shroyer AL, et al. Off-Pump coronary artery bypass surgery is associated with worse arterial and saphenous vein graft patency and less effective revascularization: Results from the Veterans Affairs Randomized On/Off Bypass (ROOBY) trial. Circulation 2012;125:2827-35.

13. Lamy A, Devereaux PJ, Prabhakaran D, T et al. Five-Year Outcomes after Off-Pump or On-Pump Coronary-Artery Bypass Grafting. N Engl J Med 2016;375:2359-68.

14. Ronald A, Patel A, Dunning J. Is the Allen's test adequate to safely confirm that a radial artery may be harvested for coronary arterial bypass grafting? Interact Cardiovasc Thorac Surg 2005;4:332-40.

15. Kwak YL, Oh YJ, Kim SH, et al. Efficacy of preemptive milrinone in off-pump coronary artery bypass surgery: comparison between patients with a low and normal pre-graft cardiac index. Eur J Cardiothorac Surg 2004;26:687-93.

16. Na S, Oh YJ, Shim YH, et al. Effects of milrinone on blood flow of the Y-graft composed with the radial and the internal thoracic artery in patients with coronary artery disease. Eur J Cardiothorac Surg 2006;30:324-8.

Cite this article as: Ramponi F, Seco M, Edelman JB, Sherrah AG, Bannon PG, Brereton RJ, Wilson MK, Vallely MP. Dual inflow, total-arterial, anaortic, off-pump coronary artery bypass grafting: how to do it. Ann Cardiothorac Surg 2018;7(4):552560. doi: 10.21037/acs.2018.06.17
17. Kwak YL, Oh YJ, Shinn HK, et al. Haemodynamic effects of a milrinone infusion without a bolus in patients undergoing off-pump coronary artery bypass graft surgery. Anaesthesia 2004;59:324-31.

18. Lev-Ran O, Paz Y, Pevni D, et al. Bilateral internal thoracic artery grafting: midterm results of composite versus in situ crossover graft. Ann Thorac Surg 2002;74:704-10; discussion 710-1.

19. Benedetto U, Altman DG, Gerry S, et al. Pedicled and skeletonized single and bilateral internal thoracic artery grafts and the incidence of sternal wound complications: Insights from the Arterial Revascularization Trial. J Thorac Cardiovasc Surg 2016;152:270-6.

20. Kim R, Baumgartner N, Clements J. Routine left atrial appendage ligation during cardiac surgery may prevent postoperative atrial fibrillation-related cerebrovascular accident. J Thorac Cardiovasc Surg 2013;145:582-9; discussion 589.

21. Ross DE. A novel custom-made long shunt simplifies the performance and improves the results of beating-heart surgery. Heart Surg Forum 2003;6:E191-3.

22. Amin S, Pinho-Gomes AC, Taggart DP. Relationship of Intraoperative Transit Time Flowmetry Findings to Angiographic Graft Patency at Follow-Up. Ann Thorac Surg 2016;101:1996-2006.

23. Edelman JJ, Reddel CJ, Kritharides L, et al. Natural history of hypercoagulability in patients undergoing coronary revascularization and effect of preoperative myocardial infarction. J Thorac Cardiovasc Surg 2014;148:536-43.

24. Royse C, Royse A, Blake D, et al. Screening the thoracic aorta for atheroma: a comparison of manual palpation, transesophageal and epiaortic ultrasonography. Ann Thorac Cardiovasc Surg 1998;4:347-50. 